\title{
LIBERAÇÃO DE NITRATO DE HIDRÓXIDOS DUPLOS LAMELARES COMO POTENCIAIS FERTILIZANTES DE LIBERAÇÃO LENTA ${ }^{(1)}$
}

\author{
Valentim da Silva ${ }^{(2)}$, Antonio Salvio Mangrich ${ }^{(3)} \&$ Fernando Wypych $^{(2)}$
}

\begin{abstract}
RESUMO
Hidróxidos duplos lamelares (HDL) são intercaladores potenciais aniônicos. Com o objetivo de obter fertilizantes de liberação lenta de nitrato, foram sintetizados HDL com a fórmula geral $\left[\mathrm{M}^{2+}{ }_{1-\mathrm{x}} \mathrm{M}^{3+}{ }_{\mathrm{x}}(\mathrm{OH})_{2}\right]^{\mathrm{x}-}\left(\mathrm{NO}_{3}\right)_{\mathrm{x}} \cdot \mathrm{yH}_{2} \mathrm{O}$, em que $\mathrm{M}^{2+}=\mathrm{Mg}^{2+} \mathrm{e}$ $\mathrm{M}^{3+}=\mathrm{Al}^{3+} \mathrm{e}$, ou, $\mathrm{Fe}^{3+}$, utilizando-se o método de coprecipitação a $\mathrm{pH}$ alcalino constante. Medidas de difração de raios-X evidenciaram que a cristalinidade aumenta com o acréscimo do valor de $\mathbf{p H}$ e da razão molar $\mathrm{M}^{2+} / \mathbf{M}^{3+}$. As medidas de FTIR apresentaram bandas características de nitrato livre no espaço interlamelar, além de pequena contaminação de íons carbonato. Medidas de TGA/DTA possibilitaram a confirmação das composições e dos teores de íons de nitrato intercalados. As curvas de liberação de nitrato demonstraram dois comportamentos de liberação complementar, um inicial rápido $(A)$ e um lento gradativo $(D)$, sendo, este último, relacionado à orientação planar do íon nitrato intercalado. Os materiais sintetizados e investigados neste trabalho apontaram grande potencial para serem utilizados como matrizes para fertilizantes de liberação lenta de nitrato.
\end{abstract}

Termos de indexação: reações de troca aniônica, barreira iônica interlamelar, coprecipitação.

\section{SUMMARY: NITRATE RELEASE FROM LAYERED DOUBLE HYDROXIDES AS POTENTIAL SLOW-RELEASE FERTILIZERS}

Layered double hydroxides ( $\mathrm{LDHs}$ ) are potential anionic intercalators. To obtain nitrate slow-release fertilizers, $\mathrm{LDHs}$ with the general formula $\left[\mathrm{M}^{2+}{ }_{1-x} \mathrm{M}^{3+}{ }_{x}(\mathrm{OH})_{2}\right]^{x-}\left(\mathrm{NO}_{3}\right)_{x} \cdot y \mathrm{H}_{2} \mathrm{O}$ (where $M^{2+}=M^{2+}$ and $M^{3+}=A l^{3+}$ and / or Fe ${ }^{3+}$ ) were synthesized by the method of co-precipitation at constant alkaline $\mathrm{pH}$. The X-ray diffraction (XRD) measurements showed that crystallinity

(1) Recebido para publicação em 24 de setembro de 2012 e aprovado em 5 de setembro de 2013.

(2) Professor, Laboratório de Química de Materiais Avançados - LAQMA, Departamento de Química, Universidade Federal do Paraná - DQ/UFPR. Caixa Postal 19081. CEP 81531-990 Curitiba (PR). E-mail: valentimdasilva@gmail.com.br, wypych@ufpr.br

(3) Professor, Laboratório de Projetos e Processos Ambientais, DQ/UFPR. E-mail: mangrich@ufpr.br 


\begin{abstract}
increases with the $\mathrm{pH}$ value and with the $\mathrm{M}^{2+} / \mathrm{M}^{3+}$ molar ratio. The FTIR measurements showed characteristic bands of free nitrate in the interlayer, as well as minor carbonate ion contamination. The compositions and concentrations of intercalated nitrate ions were confirmed by TGA/DTA measurements. The nitrate release curves showed two patterns of complementary release - fast initial release (A) and slow gradual release (D). The latter is related to the planar orientation of the intercalated nitrate ion. The synthesized materials investigated in this study proved to be highly promising as matrices for nitrate slow-release fertilizers.
\end{abstract}

Index terms: anion exchange reactions, ionic interlayer barrier, co-precipitation.

\section{INTRODUÇÃO}

Nos últimos anos, intensificaram-se as pesquisas utilizando os hidróxidos duplos lamelares (HDL) (Komarneni et al., 2003; Torres-Dorante et al., 2009; Ureña-Amate et al., 2011), com o propósito de uso na preparação de fertilizantes de liberação lenta de nitrato (Silva et al., 2014). Essas estruturas são fundamentadas na brucita $\left[\mathrm{Mg}(\mathrm{OH})_{2}\right]$, em que parte dos cátions $\mathrm{Mg}^{2+}$, coordenados octaedricamente a íons hidroxila, é substituída isomorficamente por cátions $\mathrm{M}^{3+}$ (Crepaldi \& Valim, 1998). Esse mecanismo gera compostos com fórmula genérica do tipo $\left[\mathrm{M}^{2+}{ }_{1-\mathrm{x}}\right.$ $\left.\mathrm{M}^{3+}{ }_{x}(\mathrm{OH})_{2}\right]^{x-}\left(\mathrm{A}^{-n}\right)_{x / n} \cdot \mathrm{yH}_{2} \mathrm{O}$, em que $\mathrm{M}^{2+}$ e $\mathrm{M}^{3+}$ representam, respectivamente, os cátion divalentes e trivalentes da lamela, e $\mathrm{A}^{-n} \cdot \mathrm{yH}_{2} \mathrm{O}$ representa o ânion hidratado intercalado (Wypych \& Arízaga, 2005).

Em razão da ampla diversidade na composição estrutural, os HDL podem ser sintetizados, gerando inúmeros compostos, com variedades de metais $\mathrm{M}^{2+} \mathrm{e}$ $\mathrm{M}^{3+}$ (Woo et al., 2011), e diversificação dos ânions intercalados (Tsujimura et al., 2007). São vários os métodos para obter os HDL (Crepaldi \& Valim, 1998), e o da coprecipitação a $\mathrm{pH}$ alcalino constante é um dos mais utilizados (Olanrewaju et al., 2000; Olfs et al., 2009; Chitrakar et al., 2011). Ao aplicar um método em determinadas condições de síntese e escolher os íons a serem intercalados buscam-se obter estruturas com propriedades químicas e físicas específicas. Assim, neste trabalho avaliou-se a influência da relação molar entre $\mathrm{Mg}^{2+} \mathrm{e}$ íons $\mathrm{Al}^{3+} \mathrm{e}$ $\mathrm{Fe}^{3+}$ e do $\mathrm{pH}$ no meio reacional, nas características estruturais (ou cristalográficas) e na capacidade de retenção e liberação de $\mathrm{NO}_{3}{ }^{-}$dos $\mathrm{HDL}$ sintetizados artificialmente.

\section{MATERIAL E MÉTODOS}

\section{Sínteses dos HDL intercalados com íons nitrato}

O procedimento foi adaptado de Arizaga et al. (2007), Hosni \& Srasra (2008) e Marangoni et al. (2009). Para as sínteses de obtenção dos HDL pelo método da coprecipitação em $\mathrm{pH}$ alcalino constante foram utilizados sais de nitrato $\left(\mathrm{NO}_{3}^{-}\right)$nas quantidades molares e nos valores de $\mathrm{pH}$ indicados no quadro 1.

Os sais foram dissolvidos em $50 \mathrm{~mL}$ de água destilada e desaerada, para evitar contaminações com íons carbonato presentes na água; os volumes deles foram completados para $125 \mathrm{~mL}$. Em seguida, as soluções contendo os sais foram gotejadas lentamente em uma solução de $\mathrm{NaNO}_{3}$, que continha quatro vezes a razão estequiométrica de $\mathrm{NO}_{3}$ - requerida para a relação proposta. Para o controle do $\mathrm{pH}$ gotejou-se simultaneamente uma solução de $\mathrm{NaOH} 0,2 \mathrm{~mol} \mathrm{~L}^{-1}$. Durante a síntese, a solução foi mantida em agitação e em atmosfera inerte de $\mathrm{N}_{2}$. Os compostos sintetizados foram retirados em frasco de resgate e lavados por cinco vezes com água destilada e centrifugados a $3.056 \mathrm{~g}$ por 12 min e depois secos em estufa a vácuo, a $60^{\circ} \mathrm{C}$ até peso constante.

\section{Caracterizações}

As análises de difração de raios-X (XRD) foram realizadas em um difratômetro Shimadzu modelo XRD6000 com tubo de raios-X de $\mathrm{Cu}(\lambda=1,5418 \AA)$, utilizando uma corrente de $30 \mathrm{~mA}$ e tensão de $40 \mathrm{kV}$. Os dados foram coletados na faixa de $3^{\circ}$ a $70^{\circ}(2 \theta)$, velocidade de $1^{\circ} \mathrm{min}^{-1}$ e passo de $0,02^{\circ}(2 \theta)$. Os espectros de infravermelho por transformada de Fourier (FTIR) foram coletados em um espectrofotômetro Bomem Michelson MB100, utilizando pastilhas de $\mathrm{KBr}$ prensadas a $10 \mathrm{t}$ e contendo $1 \%$ da amostra. As análises foram realizadas no modo de transmissão na faixa de $4.000-400 \mathrm{~cm}^{-1}$, com uma resolução de $8 \mathrm{~cm}^{-1}$ e acumulação de 32 varreduras.

As medidas de análise térmica (TGA/DTA) foram realizadas em um equipamento Mettler Toledo TGA/ S-DTA 851 E, utilizando cadinhos de platina, sob fluxo de $\mathrm{O}_{2}\left(50 \mathrm{~mL} \mathrm{~min}^{-1}\right)$ à taxa de aquecimento de $10^{\circ} \mathrm{min}^{-1}$.

\section{Teste de liberação de nitrato}

Seguindo descrição metodológica adaptada de Kamogawa \& Teixeira (2009) e apresentada por Silva et al. (2014), utilizou-se 0,125 g de cada HDL em $50 \mathrm{~mL}$ de solução de $\mathrm{NaHCO}_{3}$ tamponado em pH 6,5. A liberação de $\mathrm{NO}_{3}$ - foi analisada nos tempos de 0 ; 0,$08 ; 0,25 ; 0,50 ; 1 ; 5 ; 24 ; 60 ; 90 ; 120 ;$ e 150 h. Os teores de $\mathrm{NO}_{3}$ - durante o processo de liberação foram 
Quadro 1. Quantidades molares dos sais e do pH de síntese utilizados para obter os hidróxidos duplos lamelares (HDL) com as pretendidas relações $\mathrm{Mg}: \mathrm{Al}: \mathrm{Fe}$

\begin{tabular}{|c|c|c|c|c|c|}
\hline Amostra & $\mathrm{Mg}\left(\mathrm{NO}_{3}\right)_{2} \cdot 6 \mathrm{H}_{2} \mathrm{O}$ & $\mathrm{Al}\left(\mathrm{NO}_{3}\right)_{3} \cdot 9 \mathrm{H}_{2} \mathrm{O}$ & $\mathrm{Fe}\left(\mathrm{NO}_{3}\right)_{3} \cdot 9 \mathrm{H}_{2} \mathrm{O}$ & pH de sintese & HDL pretendido \\
\hline & & mol & & & \\
\hline 1 & $4,591 \cdot 10^{-2}$ & & $1,530.10^{-2}$ & 9,5 & HDL- $\mathrm{Mg}_{3} \mathrm{Fe}_{1}$ \\
\hline 2 & $5,194 \cdot 10^{-2}$ & & $1,299.10^{-2}$ & 9,5 & HDL- $\mathrm{Mg}_{4} \mathrm{Fe}_{1}$ \\
\hline 3 & $5,593.10^{-2}$ & & $1,119.10^{-2}$ & 9,5 & HDL- $\mathrm{Mg}_{5} \mathrm{Fe}_{1}$ \\
\hline 4 & $8,041.10^{-2}$ & $2,010.10^{-2}$ & $2,010.10^{-2}$ & 8 & HDL-Mg ${ }_{2} \mathrm{Al}_{0,5} \mathrm{Fe}_{0,5}$ \\
\hline 5 & $8,041.10^{-2}$ & $2,010.10^{-2}$ & $2,010.10^{-2}$ & 9 & HDL- $\mathrm{Mg}_{2} \mathrm{Al}_{0,5} \mathrm{Fe}_{0,5}$ \\
\hline 6 & $8,041.10^{-2}$ & $2,010.10^{-2}$ & $2,010.10^{-2}$ & 10 & HDL-Mg ${ }_{2} \mathrm{Al}_{0,5} \mathrm{Fe}_{0,5}$ \\
\hline 7 & $7,050.10^{-2}$ & $2,350.10^{-2}$ & & 10 & HDL- $\mathrm{Mg}_{3} \mathrm{Al}_{1}$ \\
\hline 8 & $7,862.10^{-2}$ & $1,965.10^{-2}$ & & 10 & HDL- $\mathrm{Mg}_{4} \mathrm{Al}_{1}$ \\
\hline 9 & $8,355.10^{-2}$ & $1,671.10^{-2}$ & & 10 & HDL- $\mathrm{Mg}_{5} \mathrm{Al}_{1}$ \\
\hline
\end{tabular}

determinados em um sistema de fluxo e injeção automática (ASIA da Ismatec, Suiça) equipado com uma bomba peristáltica de quatro canais (IS 7610) com velocidade variável de 1 a $50 \mathrm{rpm}$, válvula eletrônica de injeção da amostra (IS 7630, Rheodyne, EUA), fotômetro de LED com comprimento de onda máximo em $605 \mathrm{~nm}$ (IS 7640 3EA), cela de fluxo de vidro, volume interno de $180 \mu \mathrm{L}$ e percurso ótico de $10 \mathrm{~mm}$ e câmara de difusão gasosa com percurso de $10 \mathrm{~cm}$ distribuídos na forma de zigue-zague (sulcos de $1 \mathrm{~mm})$.

\section{Modelo matemático aplicado}

As cinéticas de liberação dos íons nitrato em solução de $\mathrm{NaHCO}_{3}$ tamponado a $\mathrm{pH} 6,5$ foram obtidas pelo modelo de Cabrera reparametrizado por Zeviani (2009), caracterizado pela equação:

$$
\left[\mathrm{NO}_{3}\right]_{l}=A\left(1-\mathrm{e}^{-\ln (2) \cdot t / V)+D . t}\right.
$$

em que: $\left[\mathrm{NO}_{3}{ }^{-}\right]_{l}=$ concentração de $\mathrm{NO}_{3}{ }^{-}$a ser liberada no tempo $t ; A=$ taxa de $\mathrm{NO}_{3}{ }^{-}$liberado no momento rápido; $t=$ tempo de liberação; $V=$ velocidade de liberação de $\mathrm{NO}_{3}$ - em função do tempo; e $D=$ taxa de $\mathrm{NO}_{3}{ }^{-}$liberado no momento lento.

Com base nas análises descritivas e gráficas, adequou-se o modelo proposto com auxílio do programa R (R Development Core Team, 2011) e da função de regressão não linear $(n l s)$, que utiliza o método de fatoração de Newton-Gauss, sendo coerente com o modelo de liberação do $\mathrm{NO}_{3}^{-}$, que consideraram duas taxas de liberação simultâneas. De acordo com este modelo, há uma taxa inicial de rápida liberação $(A)$, supostamente ligada à porção de $\mathrm{NO}_{3}{ }^{-}$presente nas estruturas que recebem ataque inicial pela alta concentração do ânion $\mathrm{HCO}_{3}$, proveniente da solução; e outra seguida de uma liberação lenta $(D)$, com declínio na taxa rápida apresentando equilíbrio cinético no sistema de liberação gradativa. A aplicação desse modelo busca considerar coeficientes associados às taxas de cada fase, com a finalidade de interpretação química do processo e da representação em curvas de liberação de $\mathrm{NO}_{3}{ }^{-}$, em razão do tempo.

\section{RESULTADOS E DISCUSSÃO}

Com a obtenção dos compostos, verificaram-se qual a relação molar mais apropriada e qual o valor de $\mathrm{pH}$ ideal para se alcançar cada combinação entre $\mathrm{Mg}^{2+}$ $\mathrm{Fe}^{3+}$ e, ou, $\mathrm{Al}^{3+}$. O propósito de sintetizar em diferentes relação molar e valor de $\mathrm{pH}$ para as combinações químicas de íons $\mathrm{Mg}^{2+} / \mathrm{Fe}^{3+}, \mathrm{Mg}^{2+} / \mathrm{Al}^{3+}: \mathrm{Fe}^{3+}{\text { e } \mathrm{Mg}^{2+} /}^{2+}$ $\mathrm{Al}^{3+}$ foi encontrar os HDL com a maior capacidade de troca aniônica (CTA) que demonstrem alto teor de $\mathrm{NO}_{3}$. intercalado e comportamento de liberação lenta em solução de excesso de íons bicarbonato.

Na figura 1, são apresentados os difratogramas de raios-X dos compostos sintetizados. Os picos foram indexados de acordo com o sistema hexagonal com simetria romboédrica (grupo espacial R-3m), frequentemente usado na literatura para sistemas de HDL (Fraccarollo et al., 2010).

De modo geral, os compostos mais cristalinos foram aqueles que envolviam o sistema $\mathrm{Mg} / \mathrm{Al}$ (Figura 1c), em que a organização estrutural se eleva com o aumento da razão molar $\mathrm{Mg} / \mathrm{Al}$. Os demais compostos se apresentam com baixa cristalinidade e no sistema $\mathrm{Mg}_{2} \mathrm{Al}_{0,5} \mathrm{Fe}_{0,5}$, obtidos em pH 8 (Figura 1b-A), observando a presença de dois picos de difração (indicados por asteriscos), característicos do $\mathrm{Al}(\mathrm{OH})_{3}$ na variedade polimórfica bayerita (JCPDS card 770250).

No quadro 2, evidenciam-se os parâmetros de rede estruturais estimados de acordo com as condições de síntese. O parâmetro de rede " $c$ " foi alcançado, utilizando-se o espaçamento basal do pico de maior ordem possível ( $d 00 \mathrm{l})$, que foi devidamente multiplicado pelo índice do pico basal usado (simetria 3R), enquanto o parâmetro de rede " $a$ " foi obtido a partir do pico (110).

As variações dos espaçamentos basais $(\Delta d)$ foram calculadas em relação à distância basal da brucita $(d=4,78 \AA$ ) e correspondem ao espaço ocupado pelos íons de $\mathrm{NO}_{3}{ }^{-}$hidratado (diâmetro ao longo do maior eixo de $4,80 \AA$ ), intercalados de forma planar (Xu \& Zeng, 2001). Ainda se observa (Quadro 2), que os 

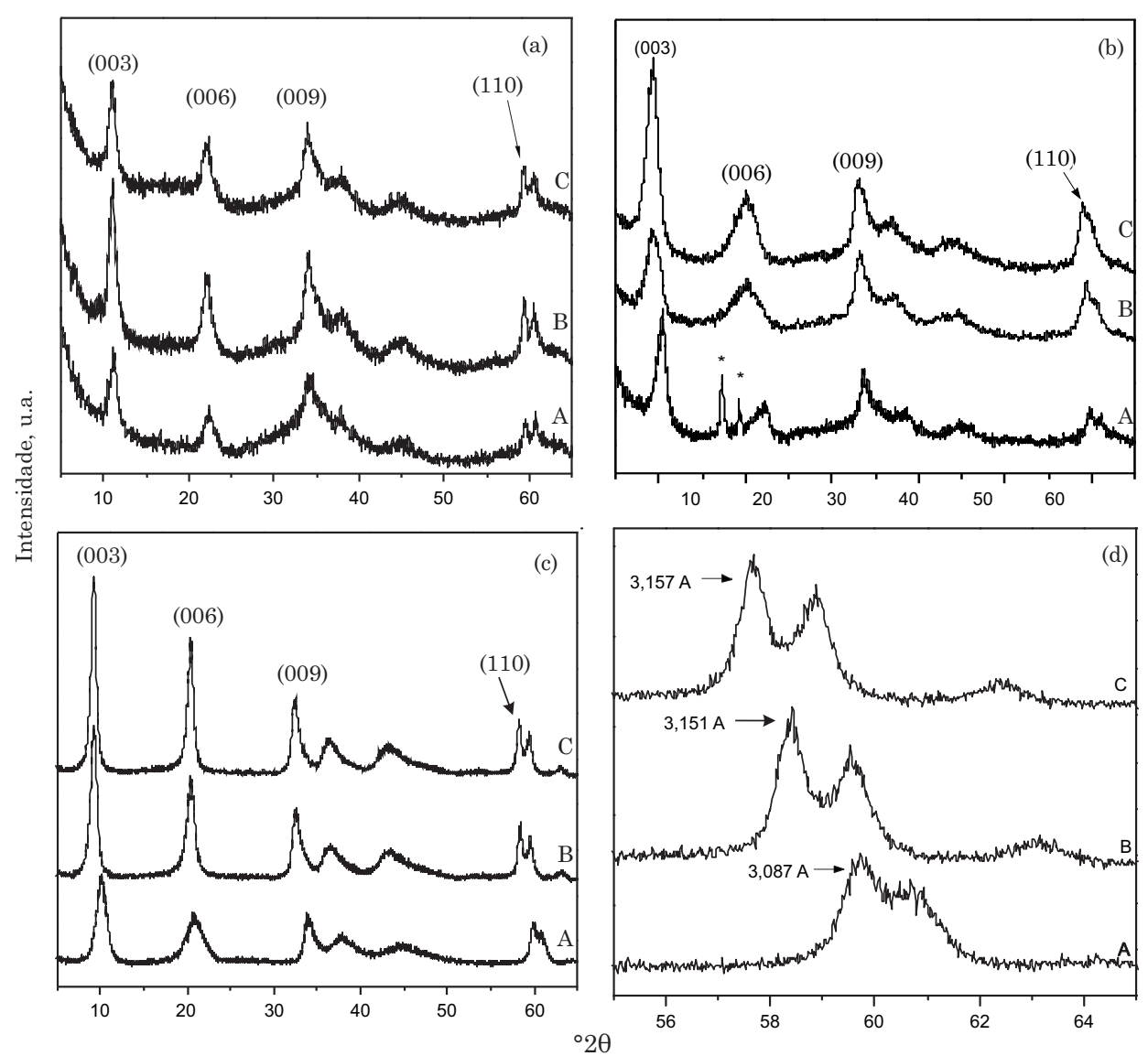

Figura 1. Difratogramas de raios-X dos compostos de hidróxido duplo lamelar (HDL): (a) - $\mathrm{HDL}-\mathrm{Mg}_{3} \mathrm{Fe}_{1}$ (A), HDL-Mg $\mathrm{Fe}_{1}$ (B) e HDL-Mg $\mathrm{Fe}_{1}$ (C), obtidos no pH 9,5; (b) - HDL-Mg $\mathrm{Al}_{0,5} \mathrm{Fe}_{0,5}$, obtido em pH 8 (A), 9 (B) e 10 (C); (c) - HDL-Mg $\mathrm{Al}_{1}$ (A), HDL-Mg $\mathrm{Ml}_{4} \mathrm{Al}_{1}$ (B) e HDL-Mg $\mathrm{Al}_{1}$ (C), obtidos no pH 10; e (d) - difratogramas expandidos do (c).

Quadro 2. Parâmetros de rede estruturais para os hidróxidos duplos lamelares (HDLs)

\begin{tabular}{|c|c|c|c|c|c|c|}
\hline Amostra & HDL-M ${ }^{2+} \mathbf{M}^{3+}$ & pH & $d 001(\AA ̊)$ & $\Delta d(\AA ̊)$ & $a(\AA)$ & $c(\AA ̊)$ \\
\hline 1 & HDL- $\mathrm{Mg}_{3} \mathrm{Fe}_{1}$ & 9,5 & 7,89 & 3,11 & 3,107 & 23,67 \\
\hline 2 & HDL- $\mathrm{Mg}_{4} \mathrm{Fe}_{1}$ & 9,5 & 7,88 & 3,10 & 3,109 & 23,64 \\
\hline 3 & HDL-Mg ${ }_{5} \mathrm{Fe}_{1}$ & 9,5 & 7,90 & 3,12 & 3,116 & 23,70 \\
\hline 4 & HDL-Mg ${ }_{02} \mathrm{Al}_{0,5} \mathrm{Fe}_{0,5}$ & 8,0 & 7,99 & 3,21 & 3,092 & 23,97 \\
\hline 5 & HDL-Mg ${ }_{2} \mathrm{Al}_{0,5} \mathrm{Fe}_{0,5}$ & 9,0 & 8,09 & 3,31 & 3,110 & 24,27 \\
\hline 6 & HDL-Mg ${ }_{2} \mathrm{Al}_{0,5} \mathrm{Fe}_{0,5}$ & 10,0 & 8,11 & 3,33 & 3,129 & 24,33 \\
\hline 7 & HDL- $\mathrm{Mg}_{3} \mathrm{Al}_{1}$ & 10,0 & 7,92 & 3,14 & 3,087 & 23,76 \\
\hline 8 & HDL-Mg $\mathrm{Mg}_{4} \mathrm{Al}_{1}$ & 10,0 & 8,20 & 3,42 & 3,151 & 24,60 \\
\hline 9 & HDL-Mg ${ }_{5} \mathrm{Al}_{1}$ & 10,0 & 8,22 & 3,44 & 3,157 & 24,66 \\
\hline
\end{tabular}

valores de $d 001$ decrescem com aumento do íon $\mathrm{M}^{3+}$, tendência atribuída ao aumento das interações eletrostáticas entre as lamelas carregadas positivamente com o $\mathrm{NO}_{3}$ - intercalado, que reduzem o espaçamento basal dos compostos. Essas interações eletrostáticas determinam a orientação dos íons no espaço interlamelar, podendo intercalá-los com orientação perpendicular, inclinado e planar. As pequenas variações nessas distâncias estão associadas ao posicionamento dos íons de $\mathrm{NO}_{3}$ - intercalado, induzidos pelas densidades de cargas positivas das lamelas. Portanto, de modo geral, estão de acordo com os valores apresentados na literatura (Xu \& Zeng, 2001; Arizaga et al., 2008).

Com relação ao parâmetro de rede " $a$ ", que mede a distância entre dois metais na lamela, os valores 
variam de acordo com o raio iônico do elemento que compõe os HDL. Um exemplo típico é apresentado na figura 1d, em que se observa o aumento do valor em razão da elevação da relação molar $\mathrm{Mg}^{2+} / \mathrm{Al}^{3+}$ na lamela, em que o maior teor de $\mathrm{Mg}^{+2}$ está associado ao aumento do valor do parâmetro de rede " $a$ ". Esse efeito é facilmente explicável por causa dos raios iônicos dos elementos utilizados, que são de 0,$66 ; 0,64$ e $0,51 \AA$ para o $\mathrm{Mg}^{2+}, \mathrm{Fe}^{3+} \mathrm{e} \mathrm{Al}^{3+}$, respectivamente.

Na figura 2, são apresentados os espectros de FTIR dos compostos sintetizados.

Em todos os espectros de FTIR são observadas bandas de 3.700 a $3.300 \mathrm{~cm}^{-1}$, referentes aos estiramentos simétricos e assimétricos dos grupamentos hidroxila, tanto de moléculas de $\mathrm{H}_{2} \mathrm{O}$ quanto de hidroxilas do retículo lamelar. Na região de $\sim 1.650-1.500 \mathrm{~cm}^{-1}$ ocorrem as vibrações de deformação angular das moléculas de $\mathrm{H}_{2} \mathrm{O}$, que estão fisisorvidas e, ou, intercaladas.

As vibrações abaixo de $600 \mathrm{~cm}^{-1}$, conhecida como região de impressão digital, são atribuídas aos estiramentos de $\mathrm{M}-\mathrm{O}$ e às flexões dos grupos $\mathrm{M}-\mathrm{O}-\mathrm{M}$, em que $\mathrm{M}=\mathrm{Mg}^{2+}, \mathrm{Al}^{3+}$ ou $\mathrm{Fe}^{3+}$. $\mathrm{Em} \sim 1.380 \mathrm{~cm}^{-1}$, são observadas vibrações correspondentes ao estiramento simétrico (v2), causado pelo $\mathrm{NO}_{3}{ }^{-}$intercalado, com alta simetria $\left(\mathrm{D}_{3 h}\right)$. Embora a reação de precipitação tenha sido conduzida em atmosfera inerte de $\mathrm{N}_{2}$, e utilizando água desaerada, a presença de carbonato intercalado é difícil de ser evitada, mesmo porque o material após secagem está exposto ao meio ambiente, o que propicia a contaminação pela adsorção de $\mathrm{CO}_{2}$ proveniente do ar atmosférico.

Ainda, para os dois compostos HDL- $\mathrm{Mg}_{2} \mathrm{Al}_{0,5} \mathrm{Fe}_{0,5}$, sintetizados em $\mathrm{pHs} 9$ e 10 , verificam-se bandas de vibração na região $\sim 1.760 \mathrm{~cm}^{-1}$, atribuídas ao $\mathrm{NO}_{3}$. Os HDL- $\mathrm{Mg}_{2} \mathrm{Al}_{0,5} \mathrm{Fe}_{0,5}$, obtidos no $\mathrm{pH} 8$, apresentam banda de vibração em $1.023 \mathrm{~cm}^{-1}$, referenciadas para o íon $\mathrm{NO}_{3}$ - com estiramento simétrico (v2). Esse composto apresenta contaminantes atribuídos à formação de bayerita $\left[\mathrm{Al}(\mathrm{OH})_{3}\right]$. Para os $\mathrm{HDL}-\mathrm{Mg}_{5} \mathrm{Al}_{1}$, constata-se a presença de bandas de vibrações em 829 e $1.763 \mathrm{~cm}^{-1}$, correspondentes aos estiramentos simétricos (v2) causados pelo $\mathrm{NO}_{3}{ }^{-}$livre intercalado com alta simetria $\left(\mathrm{D}_{3 h}\right)$. Essas bandas são provocadas pela diferente combinação estrutural dos compostos sintetizados; nesse caso, atribuído ao metal trivalente $\mathrm{Al}^{3+}$.

As curvas de análise térmica (TGA/DTA) são apresentadas na figura 3. De modo geral, o primeiro evento endotérmico na curva de DTA, próximo a $100^{\circ} \mathrm{C}$, está associado a perdas de massa nas curvas de TGA, que estão associadas à evaporação das moléculas de $\mathrm{H}_{2} \mathrm{O}$ fisisorvidas e, ou, intercaladas. Os valores crescentes para a temperatura no primeiro pico endotérmico para HDL-MgFe são atribuídos à crescente relação aos valores de $x(3: 1,4: 1$ e $5: 1)$, indicando o aumento de estabilidade desses compostos em razão da maior concentração de $\mathrm{Fe}^{3+}$ nas lamelas. Os diferentes teores de água nas amostras estão relacionados ao equilíbrio das amostras à umidade
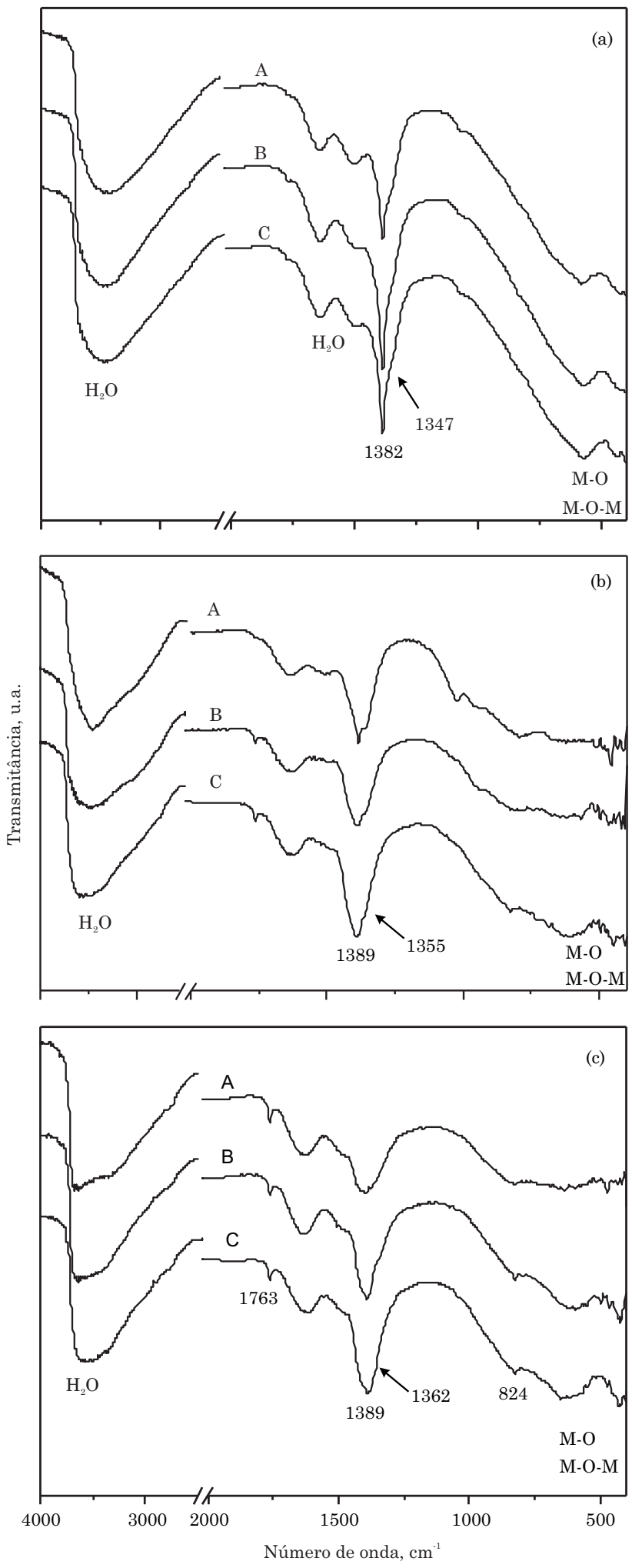

Figura 2. Espectros de FTIR dos compostos de hidróxido duplo lamelar (HDL): (a) - HDL$\mathrm{Mg}_{3} \mathrm{Fe}_{1}$ (A), HDL-Mg $\mathrm{Fe}_{1}$ (B) e HDL-Mg $\mathrm{Me}_{5}$ (C) obtidos no pH 9,5; (b) - HDL- $\mathrm{Mg}_{2} \mathrm{Al}_{0,5} \mathrm{Fe}_{0,5}$ obtido nos pHs 8 (A), 9 (B) e 10 (C); e (c) - HDL- $\mathrm{Mg}_{3} \mathrm{Al}_{1}$ (A), HDL-Mg $\mathrm{Al}_{1}$ (B) e HDL-Mg $\mathrm{Mg}_{5} \mathrm{Al}_{1}$ (C) obtido no $\mathrm{pH} 10$. 

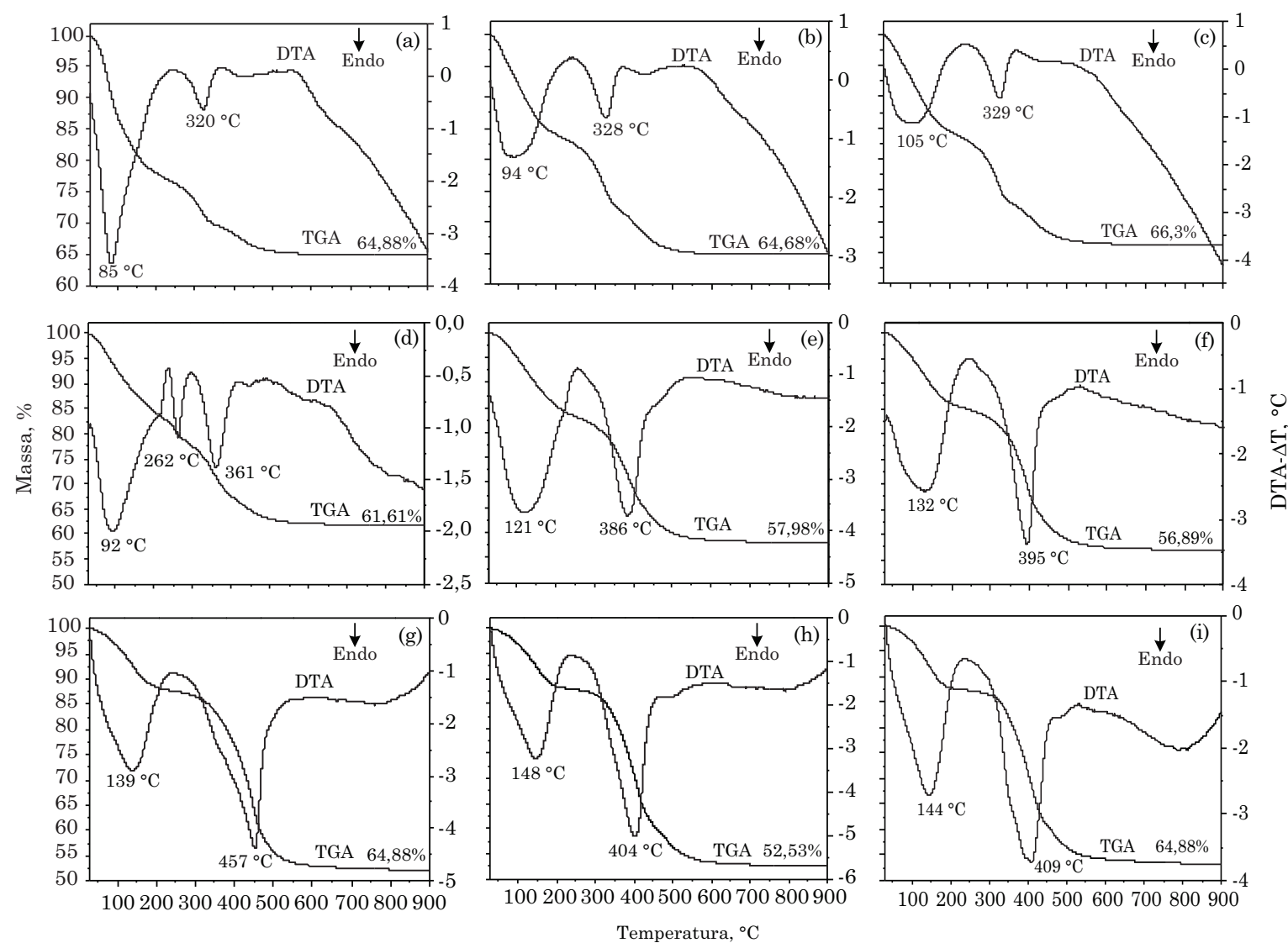

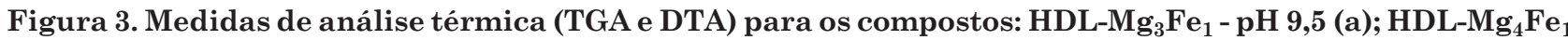
- pH 9,5 (b); HDL-Mg $\mathrm{Fe}_{1}-\mathrm{pH} \mathrm{9,5} \mathrm{(c);} \mathrm{HDL-Mg} \mathrm{Al}_{0,5} \mathrm{Fe}_{0,5}-\mathrm{pH} 8$ (d); HDL-Mg $\mathrm{Al}_{0,5} \mathrm{Fe}_{0,5}-\mathrm{pH} 9$ (e); HDL$\mathrm{Mg}_{2} \mathrm{Al}_{0,5} \mathrm{Fe}_{0,5}-\mathrm{pH} 10$ (f); HDL-Mg $\mathrm{Al}_{1}-\mathrm{pH} 10$ (g); HDL-Mg $\mathrm{Al}_{1}-\mathrm{pH} 10$ (h); e HDL-Mg $\mathrm{Al}_{1}-\mathrm{pH} 10$ (i).

relativa do ar durante estocagem antes das análises. O segundo evento endotérmico na curva DTA, acima de $350^{\circ} \mathrm{C}$, associa-se à grande perda de massa na curva de TGA, que é atribuída à desidroxilação da matriz, liberação de gases e consequente formação dos óxidos constituintes das amostras. Excepcionalmente na figura $3 \mathrm{~d}$, o comportamento térmico dos $\mathrm{HDL}-\mathrm{Mg}_{2} \mathrm{Al}_{0,5} \mathrm{Fe}_{0,5} \mathrm{pH}$ 8 apresenta três eventos endotérmicos na curva de DTA, sendo o diferencial o segundo fenômeno, atribuído à remoção de água da estrutura da bayerita.

Em razão dos teores de óxidos obtidos, foi possível estimar as fórmulas de cada composto, a capacidade de troca aniônica (CTA), a massa molar (MM) e os percentuais dos teores de $\mathrm{Mg}^{2+}, \mathrm{Fe}^{3+}, \mathrm{Al}^{3+}$ e $\mathrm{NO}_{3}{ }^{-}$ (Quadro 3).

Os HDL apresentam alta capacidade de troca aniônica (CTA), o que reflete em altos teores de ânions de $\mathrm{NO}_{3}^{-}$, que ocupam os espaços interlamelares. Esse comportamento é atribuído às substituições isomórficas de $\mathrm{M}^{2+} / \mathrm{M}^{3+}$ presentes nas estruturas das lamelas dos HDL, onde os teores de $\mathrm{M}^{+3}$ estão diretamente associados aos teores de nitrato/carbonato intercalados. Ainda, ressalta-se que as CTAs encontradas para esses HDL, de modo generalizado, são muito superiores às encontradas nos solos para argilas de alta atividade, que apresentam seu potencial máximo de capacidade de troca catiônica (CTC) com aproximadamente $150 \mathrm{cmol}_{\mathrm{c}} \mathrm{kg}^{-1}$ para os minerais como as vermiculitas e as montmorilonitas, que são pouco encontradas em solos agrícolas brasileiros (Silva et al., 2008).

Esses resultados são atribuídos não só à razão molar $\mathrm{M}^{2+} / \mathrm{M}^{3+}$, mas também à natureza dos cátions e dos ânions e ao grau de cristalinidade, um dos principais fatores que interferem no potencial de adsorção de $\mathrm{NO}_{3}^{-}$no espaço interlamelar das estruturas; e, ainda, às condições de sínteses, em que o principal objetivo não estava voltado para o aproveitamento total dos seus componentes, mas sim para ótima estruturação e desenvolvimento de CTA pela substituição dos $\mathrm{M}^{2+} / \mathrm{M}^{3+}$.

De acordo com as concentrações calculadas pelas fórmulas de HDL, sugere-se que a quantificação e os teores encontrados de $\mathrm{Mg}^{2+}, \mathrm{Al}^{3+}, \mathrm{Fe}^{3+}$ e $\mathrm{NO}_{3}$. viabilizem a utilização das estruturas para a aplicação e o fornecimento dos nutrientes para as plantas. Para a aplicação das estruturas sintetizadas como fertilizante de liberação controlada/lenta de $\mathrm{NO}_{3}{ }^{-}$, é necessário que o íon $\mathrm{NO}_{3}$ - intercalado tenha o potencial de troca pelas espécies presentes na solução do solo. 
Quadro 3. Fórmulas moleculares, capacidade de troca aniônica (CTA), massa molar (MM) e teores de íons presente nos hidróxidos duplos lamelares sintetizados

\begin{tabular}{|c|c|c|c|c|c|c|c|}
\hline Amostra & Fórmula $^{(1)}$ & CTA & MM & $\mathrm{Mg}^{2+}$ & $\mathrm{Fe}^{3+}$ & $\mathrm{Al}^{3+}$ & $\mathrm{NO}_{3}^{-}$ \\
\hline & & $\mathrm{cmol}_{\mathrm{c}} \mathrm{kg}^{-1}$ & $\mathrm{~g} \mathrm{~mol}^{-1}$ & & - & & \\
\hline 1 & $\mathrm{Mg}_{0,75} \mathrm{Fe}_{0,25}(\mathrm{OH})_{2}\left(\mathrm{NO}_{3}\right)_{0,25} \cdot 1,37 \mathrm{H}_{2} \mathrm{O}$ & 235 & 106 & 17,1 & 13,1 & & 14,6 \\
\hline 2 & $\mathrm{Mg}_{0,80} \mathrm{Fe}_{0,20}(\mathrm{OH})_{2}\left(\mathrm{NO}_{3}\right)_{0,20} \cdot 0,80 \mathrm{H}_{2} \mathrm{O}$ & 219 & 91 & 21,3 & 12,2 & & 13,6 \\
\hline 3 & $\mathrm{Mg}_{0,83} \mathrm{Fe}_{0,17}(\mathrm{OH})_{2}\left(\mathrm{NO}_{3}\right)_{0,17} \cdot 0,81 \mathrm{H}_{2} \mathrm{O}$ & 186 & 91 & 22,1 & 10,4 & & 11,5 \\
\hline 4 & $\mathrm{Mg}_{0,66} \mathrm{Al}_{0,17} \mathrm{Fe}_{0,17}(\mathrm{OH})_{2}\left(\mathrm{NO}_{3}\right)_{0,33} \cdot 0,97 \mathrm{H}_{2} \mathrm{O}$ & 323 & 102 & 15,7 & 9,3 & 4,5 & 20,0 \\
\hline 5 & $\mathrm{Mg}_{0,66} \mathrm{Al}_{0,17} \mathrm{Fe}_{0,17}(\mathrm{OH})_{2}\left(\mathrm{NO}_{3}\right)_{0,33} \cdot 0,95 \mathrm{H}_{2} \mathrm{O}$ & 325 & 102 & 15,8 & 9,3 & 4,5 & 20,1 \\
\hline 6 & $\mathrm{Mg}_{0,66} \mathrm{Al}_{0,17} \mathrm{Fe}_{0,17}(\mathrm{OH})_{2}\left(\mathrm{NO}_{3}\right)_{0,33} \cdot 0,84 \mathrm{H}_{2} \mathrm{O}$ & 331 & 100 & 16,1 & 9,5 & 4,6 & 20,5 \\
\hline 7 & $\mathrm{Mg}_{0,75} \mathrm{Al}_{0,25}(\mathrm{OH})_{2}\left(\mathrm{NO}_{3}\right)_{0,25} .0,59 \mathrm{H}_{2} \mathrm{O}$ & 294 & 85 & 21,4 & & 7,9 & 18,2 \\
\hline 8 & $\mathrm{Mg}_{0,80} \mathrm{Al}_{0,20}(\mathrm{OH})_{2}\left(\mathrm{NO}_{3}\right)_{0,20} \cdot 0,54 \mathrm{H}_{2} \mathrm{O}$ & 247 & 81 & 24,0 & & 6,7 & 15,3 \\
\hline 9 & $\mathrm{Mg}_{0,83} \mathrm{Al}_{0,17}(\mathrm{OH})_{2}\left(\mathrm{NO}_{3}\right)_{0,17} \cdot 0,56 \mathrm{H}_{2} \mathrm{O}$ & 214 & 79 & 25,4 & & 5,8 & 13,3 \\
\hline
\end{tabular}

(1) $\mathrm{M}^{2+}{ }_{1-x} \mathrm{M}^{3+}{ }_{x}(\mathrm{OH})_{2}{ }^{x-} \mathrm{A}^{-n}{ }_{x / n} \cdot \mathrm{yH}_{2} \mathrm{O}$

As estruturas de HDL sintetizadas foram submetidas a processos de liberação de $\mathrm{NO}_{3}^{-}$em solução de $\mathrm{NaHCO}_{3}$ tamponada a $\mathrm{pH} 6,5$, para observar os efeitos cinéticos de troca aniônica. Nessas condições, o $\mathrm{HCO}_{3}{ }^{-}$em solução é o íon esperado para trocar com os íons $\mathrm{NO}_{3}^{-}$das estruturas. O comportamento cinético de troca aniônica $\left(\mathrm{NO}_{3}{ }^{-} / \mathrm{HCO}_{3}{ }^{-}\right)$ e a liberação de $\mathrm{NO}_{3}{ }^{-}$na solução de $\mathrm{HCO}_{3}{ }^{-}(\mathrm{pH} 6,5)$ foram representados a partir dos valores preditos, com o intervalo de confiança (IC) de $95 \%$, em razão do tempo de $150 \mathrm{~h}$. O comportamento de troca demonstra dois momentos distintos e complementares de liberação de $\mathrm{NO}_{3}$. O primeiro momento, liberação rápida $(A)$ e, o segundo, liberação lenta $(D)$. De acordo com Zeviani (2009), por meio da equação $\left[\mathrm{NO}_{3}{ }^{-}\right]_{l}=A\left(1-\mathrm{e}^{-\ln (2) \cdot t / V}\right)$ + D.t é possível aplicar o ajuste, considerando os dois momentos integrados, $A$ e $D$. A representação numérica dos índices e do somatório dos dois momentos no total do tempo $(150 \mathrm{~h})$, respectivamente para cada HDL, submetidos ao processo de liberação de $\mathrm{NO}_{3}{ }^{-}$ em solução encontram-se no quadro 4. Essas taxas, do quadro 4, demonstram serem significativas estatisticamente.

Afirma-se, com esses resultados, que houve pouca liberação de $\mathrm{NO}_{3}{ }^{-}$no momento $A$, demonstrando que o maior percentual de $\mathrm{NO}_{3}$ - continua retido nos HDL. $\mathrm{O}$ diferencial de liberação de $\mathrm{NO}_{3}{ }^{-}$entre os $\mathrm{HDL}$, para os momentos $A$ e $D$, é atribuído principalmente à densidade de carga, ao tamanho dos cristais, à composição química da lamela e à cristalinidade, sendo a densidade de carga relativa às substituições de $\mathrm{M}^{2+}$ $\mathrm{M}^{3+}$, que determinam o teor de $\mathrm{NO}_{3}$ - intercalado nas estruturas. $\mathrm{O}$ aumento da cristalinidade sugere maior tamanho dos cristais, que permite maiores concentrações de $\mathrm{NO}_{3}{ }^{-}$intercalado. Dessa forma, o momento $A$ é a representação das trocas aniônicas de arestas e regiões periféricas dos cristais lamelares, sendo consequência dessas trocas o surgimento do efeito chamado "barreira iônica interlamelar". Esse mecanismo (Figura 4, $2^{\mathrm{a}}$ Fase) impede que os íons
$\mathrm{NO}_{3}{ }^{-}$que estão presentes na região central dos cristais sejam trocados pelos íons $\mathrm{HCO}_{3}^{-}$, em razão da barreira iônica formada nas laterais dos cristais lamelares. Também, o que contribui para o mecanismo de troca lenta (momento $D$ ) é a orientação dos íons $\mathrm{NO}_{3}$ intercalados no espaço interlamelar. A distância basal obtida (Quadro 2) não é suficiente para acomodar os íons na orientação perpendicular, de tal forma que esses íons se encontram provavelmente na orientação planar ou inclinada. Essas orientações (planar e inclinada) em conjunto com as barreiras iônicas interlamelares acentuam a retenção do $\mathrm{NO}_{3}{ }^{-}$na região central dos cristais, ocasionando trocas mais lentas e liberação gradativa do íon $\mathrm{NO}_{3}{ }^{-}$. Dessa forma, verificase que o momento $D$, em razão do tempo, é crescente ao longo das $150 \mathrm{~h}$.

Esses mecanismos caracterizam a troca lenta para essas estruturas. Ainda, o efeito da barreira iônica interlamelar está diretamente relacionado com os tamanhos dos cristais e a composição química deles. Observa-se que os compostos com presença de $\mathrm{Al}^{3+}$ liberaram mais $\mathrm{NO}_{3}$ - do que compostos com $\mathrm{Fe}^{3+}$ (Quadro 4), indicando que o $\mathrm{Fe}^{3+}$ retém $\mathrm{NO}_{3}{ }^{-}$nas estruturas com maior força de ligação e menor possibilidade de deslocamento desse ânion.

Percebe-se que para as estruturas de HDL-MgFe, os teores do momento rápido são menores do que os de $\mathrm{NO}_{3}{ }^{-}$liberados em amostras que contêm íons de $\mathrm{Al}^{3+}$. Essa relação sugere que compostos de HDL sintetizados com íons de $\mathrm{Fe}^{3+}$ têm melhores propensões de retenção de $\mathrm{NO}_{3}^{-}$, e a sua liberação de forma gradativa. Dessa forma, o comportamento de liberação rápida do $\mathrm{HDL}-\mathrm{Mg}_{4} \mathrm{Fe}_{1}$ apresenta maiores possibilidades de previsibilidade, por causa da sua melhor cristalinidade dentre os demais compostos. Também, o que se soma a essa afirmação está relacionado à taxa de liberação lenta que, embora apresente suave diferença, observa-se que é menor e ao longo do tempo no meio ambiente. Essa diferença que é gradativa pode ser significativamente positiva 
Quadro 4. Taxa de liberação rápida e lenta de $\mathrm{NO}_{3}$ - hidróxidos duplos lamelares (HDL) com diferetnes relações Mg:Al:Fe em solução de $\mathrm{HCO}_{3}^{-}$, em $150 \mathrm{~h}$

\begin{tabular}{|c|c|c|c|c|c|c|}
\hline Amostra & Fórmula & $A^{(1)}$ & $\mathrm{D}^{(1)}$ & $\mathrm{V}^{(1)}$ & Tmv & $\mathrm{NO}_{3}^{-}$ \\
\hline & & & & & $\mathrm{h}$ & $\%$ total \\
\hline 1 & $\mathrm{Mg}_{0,75} \mathrm{Fe}_{0,25}(\mathrm{OH})_{2}\left(\mathrm{NO}_{3}\right)_{0,25} \cdot 1,37 \mathrm{H}_{2} \mathrm{O}$ & 8,1997 & 0,536 & 0,039 & 67,33 & 88,6 \\
\hline 2 & $\mathrm{Mg}_{0,80} \mathrm{Fe}_{0,20}(\mathrm{OH})_{2}\left(\mathrm{NO}_{3}\right)_{0,20} \cdot 0,80 \mathrm{H}_{2} \mathrm{O}$ & 15,965 & 0,534 & 0,202 & 59,82 & 96,1 \\
\hline 3 & $\mathrm{Mg}_{0,83} \mathrm{Fe}_{0,17}(\mathrm{OH})_{2}\left(\mathrm{NO}_{3}\right)_{0,17} \cdot 0,81 \mathrm{H}_{2} \mathrm{O}$ & 14,994 & 0,559 & 0,346 & 61,32 & 98,8 \\
\hline 4 & $\mathrm{Mg}_{0,66} \mathrm{Al}_{0,17} \mathrm{Fe}_{0,17}(\mathrm{OH})_{2}\left(\mathrm{NO}_{3}\right)_{0,33} \cdot 0,97 \mathrm{H}_{2} \mathrm{O}$ & 13,572 & 0,573 & 0,046 & 63,13 & 99,6 \\
\hline 5 & $\mathrm{Mg}_{0,66} \mathrm{Al}_{0,17} \mathrm{Fe}_{0,17}(\mathrm{OH})_{2}\left(\mathrm{NO}_{3}\right)_{0,33} \cdot 0,95 \mathrm{H}_{2} \mathrm{O}$ & 21,422 & 0,548 & 0,202 & 55,31 & 103,6 \\
\hline 6 & $\mathrm{Mg}_{0,66} \mathrm{Al}_{0,17} \mathrm{Fe}_{0,17}(\mathrm{OH})_{2}\left(\mathrm{NO}_{3}\right)_{0,33} \cdot 0,84 \mathrm{H}_{2} \mathrm{O}$ & 26,023 & 0,504 & 0,389 & 49,00 & 101,6 \\
\hline 7 & $\mathrm{Mg}_{0,75} \mathrm{Al}_{0,25}(\mathrm{OH})_{2}\left(\mathrm{NO}_{3}\right)_{0,25} .0,59 \mathrm{H}_{2} \mathrm{O}$ & 41,608 & 0,399 & 0,554 & 22,55 & 101,4 \\
\hline 8 & $\mathrm{Mg}_{0,80} \mathrm{Al}_{0,20}(\mathrm{OH})_{2}\left(\mathrm{NO}_{3}\right)_{0,20} \cdot 0,54 \mathrm{H}_{2} \mathrm{O}$ & 33,852 & 0,457 & 0,216 & 37,88 & 102,4 \\
\hline 9 & $\mathrm{Mg}_{0,83} \mathrm{Al}_{0,17}(\mathrm{OH})_{2}\left(\mathrm{NO}_{3}\right)_{0,17} \cdot 0,56 \mathrm{H}_{2} \mathrm{O}$ & 30,099 & 0,494 & 0,087 & 44,49 & 104,2 \\
\hline
\end{tabular}

(1) $\left[\mathrm{NO}_{3}{ }^{-}\right]_{1}=\mathrm{A}\left(1-\mathrm{e}^{-\ln (2) . t / \mathrm{V}}\right)+$ D.t, em que $\left[\mathrm{NO}_{3}{ }^{-}\right]_{1}=$ concentração de $\mathrm{NO}_{3}{ }^{-}$a ser liberada no tempo $t$; $\mathrm{A}=$ taxa de liberação de nitrato no momento rápido; $\mathrm{D}=$ taxa de liberação de nitrato no momento lento; $\mathrm{V}$ = velocidade de liberação de nitrato em razão do tempo; Tmv: tempo necessário para que seja liberado $50 \%$ do nitrato total presente na solução.

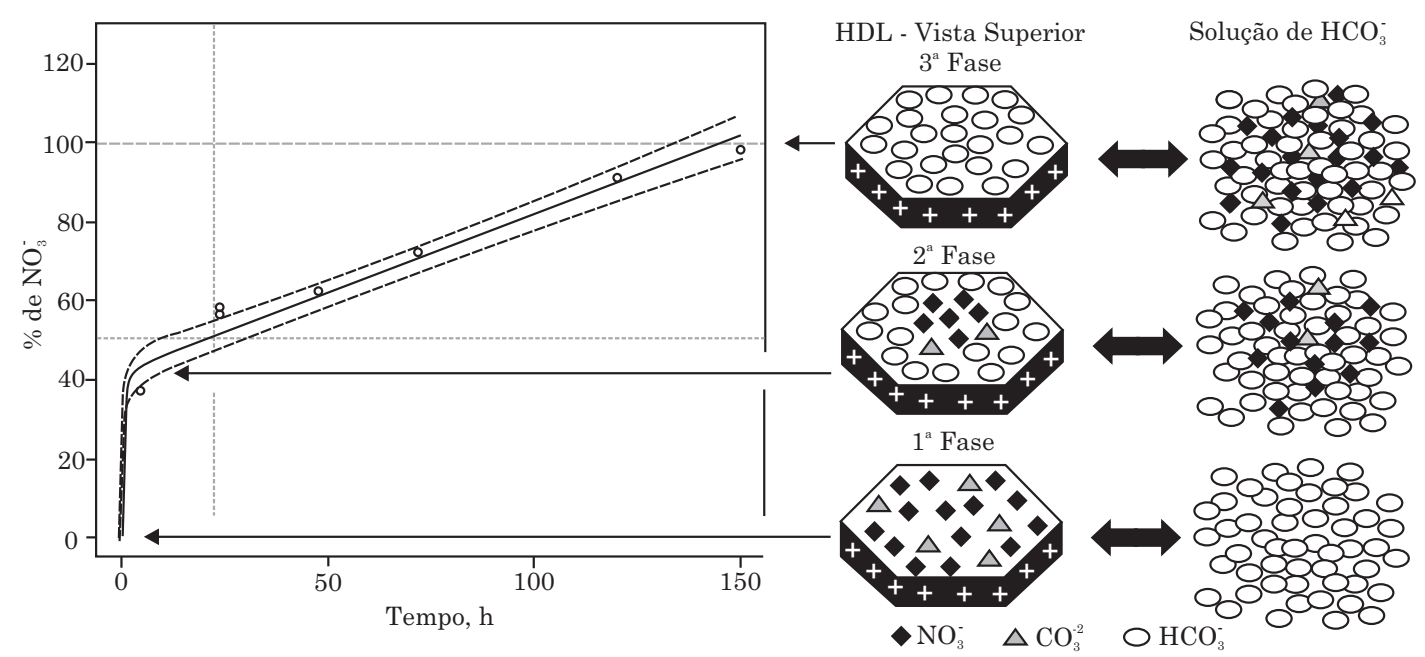

Figura 4. Representação das trocas aniônicas de arestas e regiões periféricas dos cristais e surgimento do efeito chamado "barreira iônica interlamelar" ( $2^{\mathrm{a}}$ Fase).

para que essas estruturas tenham seu maior potencial em utilizações como matrizes para fertilizantes de liberação lenta de nitrato.

Comparativamente, o valor do Tmv é diretamente influenciado pela presença de $\mathrm{Fe}^{3+}$ nas estruturas de HDL (Quadro 4), indicando que houve maior força de retenção de $\mathrm{NO}_{3}{ }^{-}$, restringindo as trocas aniônicas no momento $A$. Isso demonstra que o maior peso atômico, os campos de orbitais e a densidade de carga em relação ao raio iônico do $\mathrm{Fe}^{3+}(0,64 \AA)$ pelo $\mathrm{Al}^{3+}(0,51 \AA)$ em substituição isomórfica pelo $\mathrm{Mg}^{2+}(0,66 \AA)$ favoreceram a retenção de $\mathrm{NO}_{3}^{-}$, prontamente liberável $(A)$ nas estruturas de HDL-MgFe. Ainda, o parâmetro de rede basal apresenta-se menor para o HDL-Mg $\mathrm{Fe}_{1}$ $(c=23,85 \AA)$ do que para o HDL- $\mathrm{Mg}_{5} \mathrm{Al}_{1}(c=24,57 \AA)$, sendo a diferença da distância dos espaços interlamelares $(\Delta d)$ para o HDL- $\mathrm{Mg}_{4} \mathrm{Fe}_{1} \Delta d=3,17 \AA$ e para o HDL-Mg $\mathrm{Mg}_{5} \mathrm{Al}_{1} \Delta d=3,41 \AA$.
As estruturas de HDL-MgFe evidenciam somente uma banda vibracional para o $\mathrm{NO}_{3}{ }^{-}$livre, ao contrário de HDL-MgAlFe e HDL-MgAl, que apresentam duas e três bandas vibracionais de $\mathrm{NO}_{3}-\mathrm{O}$ aumento nas bandas de vibrações atribuídas ao $\mathrm{NO}_{3}$ - indica as diferentes coordenações possíveis entre os arranjos dos octaedros nas lamelas das estruturas de HDL. O íon $\mathrm{NO}_{3}$ - intercalado pode estar numa "área" de octaedros formados por $3 \mathrm{M}^{2+}, 2 \mathrm{M}^{2+}+\mathrm{M}^{3+}, \mathrm{M}^{2+}+2 \mathrm{M}^{3+}$ e $3 \mathrm{M}^{3+}$, implicando na não homogeneidade da estrutura da lamela, o que, em algum momento, favorece ou restringe a liberação de $\mathrm{NO}_{3}{ }^{-}$. As estruturas que apresentam $3 \mathrm{M}^{2+}$ podem ter pontos "neutros" na lamela, o que facilitaria a troca com íon intercalado; nesse caso, sugerido para as estruturas que contêm o metal $\mathrm{Al}^{3+}$, o que justificaria a maior liberação no momento $A$ de $\mathrm{NO}_{3}$. Esses fatores são determinantes, pois apontam para que a estrutura $\mathrm{HDL}-\mathrm{Mg}_{4} \mathrm{Fe}_{1}$ tenha 
o maior potencial para ser utilizada como matriz de fertilizantes de liberação lenta de $\mathrm{NO}_{3}$.

A menor CTA é proporcionada pelos menores teores de $\mathrm{M}^{3+}$ na lamela, ocasionando redução dos teores de $\mathrm{NO}_{3}$ - intercalado e consequentemente aumento da distância basal. Esse conjunto de fenômenos relacionase diretamente com a disposição dos íons no espaço interlamelar; o $\mathrm{NO}_{3}{ }^{-}$livre $\left(D_{3 h}\right)$ pode se alojar de três formas: "plana", em que os planos moleculares dos íons estão paralelamente com as lamelas das estruturas, fenômeno que ocorre quando as lamelas apresentam menor densidade de carga; "inclinada", quando há aumento de carga na lamela e na concentração de $\mathrm{NO}_{3}{ }^{-}$nos espaços interlamelares, mas a distância interlamelar ainda não é capaz para acomodar o íon na vertical e as forças eletrostáticas das lamelas não são suficientemente fortes para romper a repulsão entre os íons de $\mathrm{NO}_{3}{ }^{-}$, favorecendo esse tipo de disposição; e "perpendicular", em que essa disposição iônica interlamelar ocorre quando as cargas positivas das lamelas se elevam e quebram a repulsão que ocorre entre os ânions, aumentando o espaçamento basal que proporciona maior concentração de íons nos espaços interlamelares.

\section{CONCLUSÕES}

1. A cristalinidade e o tamanho dos cristais dos HDL são influenciados pelos tipos de metais trivalentes presentes nas lamelas.

2. A cristalinidade dos HDL sintetizados no $\mathrm{pH} 10$ aumentou com a elevação da relação $\mathrm{Mg}^{2+} / \mathrm{Al}^{3+}$.

3. As estruturas com a presença dos íons $\mathrm{Fe}^{3+} \mathrm{e}$ $\mathrm{Al}^{3+}$ na lamela apresentaram baixa cristalinidade.

4. Os HDL com menores cristais liberam mais nitrato no momento rápido e menos no lento.

5. O diferencial de liberação no momento rápido entre os HDL sintetizados dependeu, principalmente, dos íons presentes nas lamelas; a liberação de $\mathrm{NO}_{3}$. aumentou com a presença de $\mathrm{Al}^{3+}$.

6. Há menor liberação no momento rápido em razão do aumento da cristalinidade para os HDL-MgAl.

7. Os HDL-MgFe apresentaram, significativamente, maior retenção de nitrato, tanto para o momento rápido quanto para o lento, evidenciando a maior afinidade do íon $\mathrm{NO}_{3}^{-}$pelo íon $\mathrm{Fe}^{3+}$, independentemente da cristalinidade.

8. A contaminação com o íon $\mathrm{CO}_{3}{ }^{2-}$ no espaço interlamelar favorece a cristalinidade dos HDL sintetizados.

9. Em razão da facilidade de síntese, os HDL têm potencial para a produção de fertilizantes de liberação lenta de $\mathrm{NO}_{3}$. Esses materiais, em caso de desestruturação, podem fornecer nutrientes complementares e essenciais às plantas, como $\mathrm{Mg}^{2+} \mathrm{e} \mathrm{Fe}^{3+}$.

\section{LITERATURA CITADA}

ARIZAGA, G.G.C.; SATYANARAYANA, K.G. \& WYPYCH, F. Layered hydroxide salts: Synthesis, properties and potential applications. Solid State Ionics, 178:1143-1162, 2007.

ARIZAGA, G.G.C.; MANGRICH, A.S.; GARDOLINSKI, J.E.F.C. \& WYPYCH, F. Chemical modification of zinc hydroxide nitrate and Zn-Al-layered double hydroxide with dicarboxylic acids. J. Colloid Interf. Sci., 320:168176, 2008.

CHITRAKAR, R.; MAKITA, Y.; SONODA, A. \& HIROTSU, T. Fe-Al layered double hydroxide in bromated reduction: Synthesis and reactivity. J. Colloid Interf. Sci., 354:798803, 2011.

CREPALDI, E.L. \& VALIM, J.B. Hidróxidos duplos lamelares: Síntese, estrutura, propriedades e aplicações. Quím. Nova, 21:300-311, 1998.

FRACCAROLlo, A.; COSSI, M. \& MARCHESE, L. DFT simulation of $\mathrm{Mg} / \mathrm{Al}$ hydrotalcite with different intercalated anions: Periodic structure and solvating effects on the odide/triiodide redox couple. Chem. Phys. Lett., 494:274-278, 2010.

HOSNI, K. \& SRASRA, E. Nitrate adsorption from aqueous solution by $\mathrm{M}^{\mathrm{II}}-\mathrm{Al}-\mathrm{CO}_{3}$ layered double hydroxide. Inorg. Mater., 44:742-749, 2008

KAMOGAWA, M.Y. \& TEIXEIRA, M.A. Autoamostrador de baixo custo para análise por injeção em fluxo. Quím. Nova, 32:1644-1646, 2009.

KOMARNENI, S.; NEWALKAR, B.; LI, D.; GHEYI, T.; LOPANO, C.; HEANEY, P. \& POST, J. Anionic clays as potential slow-release fertilizers: Nitrate ion exchange. J. Porous Mat., 10:243-248, 2003.

MARANGONI, R.; BOUHENT, M.; TAVIOT-GUEHO, C.; WYPYCH, F. \& LEROUXC, F. $\mathrm{Zn}_{2} \mathrm{Al}$ layered double hydroxides intercalated and adsorbed with anionic blue dyes: A physico-chemical characterization. J. Colloid Interf. Sci., 333:120-127, 2009.

OLANREWAJU, J.; NEWALKAR, B.L.; MANCINO, C. \& KOMARNENI, S. Simplified synthesis of nitrate form of layered double hydroxide. Mater. Lett., 45:307-310, 2000.

OLFS, H.W.; TORRES-DORANTE, L.O.; ECKELT, R. \& KOSSLICK, H. Comparison of different synthesis for $\mathrm{Mg}-\mathrm{Al}$ layered double hydroxides ( $\mathrm{LDH})$ : Characterization of the structural phases and anion exchange properties. Appl. Clay Sci., 43:459-464, 2009.

R DEVELOPMENT CORE TEAM. R: A language and environment for statistical computing. R Foundation for Statistical Computing. Vienna, 2011. ISBN 3-90005107-0, 2011. Disponível em: <http://www.R-project.org>. Acesso em: 24 out. 2011.

SILVA, V.; MOTTA, A.C.V.; MELO, V.F. \& LIMA, V.C. Variáveis de acidez em função da mineralogia da fração argila do solo. R. Bras. Ci. Solo, 32:551-559, 2008. 
SILVA, V.; KAMOGAWA, M.; MARANGONI, R.; MANGRICH, A.S. \& WYPYCH, F. Hidróxidos duplos lamelares como matrizes para fertilizantes de liberação lenta de nitrato, R. Bras. Ci Solo. 38:272-277, 2014

TORRES-DORANTE, L.O.; LAMMEL, J. \& KUHLMANN, H. Use of a layered double hydroxide (LDH) to buffer nitrate in soil: Long-term nitrate exchange properties under cropping and fallow conditions. Plant Soil, 315:257-272, 2009.

TSUJIMURA, A.; UCHIDA, M. \& OKUWAKI, A. Synthesis and sulfate ion-exchange properties of a hydrotalcitelike compound intercalated by chloride ions. J. Hazard. Mater., 143:582-586, 2007.

UREÑA-AMATE, M.D.; BOUTARBOUCH, N.D.; SOCIASVICÍANA, M.M. \& GONZÁLES-PRADAS, E. Controlled release of nitrate from hydrotalcite modified formulations. Appl. Clay Sci., 52:368-373, 2011.
WOO, M.A.; KIM, T.W.; PAEK, M.; HA, H.; CHOY, J. \& HWANG, S. Phosphate-intercalated Ca-Fe-layered double hydroxides: Crystal structure, bonding character, and release kinects of phosphate. J. Solid State Chem., 184:171-176, 2011.

WYPYCH, F. \& ARÍZAGA, G.G.C. Intercalação e funcionalização da brucita com ácidos carboxílicos. Quím. Nova, 28:24-29, 2005.

XU, Z.P. \& ZENG, H.C. Abrupt structural transformation in hidrotalcite-like compounds $\mathrm{Mg}_{1-x} \mathrm{Al}_{x}(\mathrm{OH})_{2}\left(\mathrm{NO}_{3}\right)_{x} \cdot n \mathrm{H}_{2} \mathrm{O}$ as a continuous function of nitrate anions. J. Phys. Chem., 105:1743-1749, 2001.

ZEVIANI, W.M. Avaliação de modelos de regressão não linear na cinética de liberação de potássio de resíduos orgânicos. Lavras, Universidade Federal de Lavras, 2009. 85p. (Dissertação de Mestrado) 